Management international

International Management

Gestiòn Internacional

Le rôle des différentes proximités pour construire des relations d'affaires dans un cluster. Le cas du SPL Mecanic Vallée

The Role of Different Proximities to Build Business Relationships

in a Cluster: the Case of the Local Productive System: Mecanic

Vallée

El papel de las diferentes proximidades en la construcción de relaciones comerciales en un clúster. El caso del SPL "Mecanic Vallée”

Bastien Bezzon et Rachel Levy

Volume 24, numéro 1, 2020

URI : https://id.erudit.org/iderudit/1069093ar

DOI : https://doi.org/10.7202/1069093ar

Aller au sommaire du numéro

Éditeur(s)

HEC Montréal

Université Paris Dauphine

ISSN

1206-1697 (imprimé)

1918-9222 (numérique)

Découvrir la revue

Citer cet article

Bezzon, B. \& Levy, R. (2020). Le rôle des différentes proximités pour construire des relations d'affaires dans un cluster. Le cas du SPL Mecanic Vallée.

Management international / International Management / Gestiòn Internacional, 24(1), 43-55. https://doi.org/10.7202/1069093ar

\section{Résumé de l'article}

Notre proposition cherche à étudier l'impact des différentes formes de proximités (géographique, organisationnelle, cognitive et sociale) pour expliquer l'existence de relations d'affaires au sein d'un cluster. Une attention particulière est portée à l'influence de la proximité sociale via les relations personnelles pour expliquer l'émergence des relations d'affaires. Nous proposerons une étude du Système Productif Local Mecanic Vallée, basée sur une méthode mixte proposant des résultats quantitatifs issus d'un modèle logit binaire, complété par des études de cas qualitatives.
Tous droits réservés (C Management international / International Management / Gestión Internacional, 2020
Ce document est protégé par la loi sur le droit d'auteur. L’utilisation des services d’Érudit (y compris la reproduction) est assujettie à sa politique d'utilisation que vous pouvez consulter en ligne.

https://apropos.erudit.org/fr/usagers/politique-dutilisation/ 


\title{
Le rôle des différentes proximités pour construire des relations d'affaires dans un cluster. Le cas du SPL Mecanic Vallée $e^{\star}$
}

\author{
The Role of Different Proximities to Build Business Relationships \\ in a Cluster: the Case of the Local Productive System: Mecanic Vallée
}

\section{El papel de las diferentes proximidades en la construcción de relaciones comerciales en un clúster. El caso del SPL "Mecanic Vallée"}

\author{
BASTIEN BEZZON \\ LEREPS, Université de Bordeaux, Université de Toulouse
}

\author{
RACHEL LEVY \\ LEREPS, ENSFEA, Université de Toulouse
}

\section{RÉSUMÉ}

Notre proposition cherche à étudier l'impact des différentes formes de proximités (géographique, organisationnelle, cognitive et sociale) pour expliquer l'existence de relations d'affaires au sein d'un cluster. Une attention particulière est portée à l'influence de la proximité sociale via les relations personnelles pour expliquer l'émergence des relations d'affaires. Nous proposerons une étude du Système Productif Local Mecanic Vallée, basée sur une méthode mixte proposant des résultats quantitatifs issus d'un modèle logit binaire, complété par des études de cas qualitatives.

Mots-Clés : Proximités, relations d'affaire, cluster, méthode mixte

\section{ABSTRACT}

Our article aim to study the impact of different forms of proximity (geographic, organizational, cognitive and social) in order to explain the existence of business relationship inside a cluster. A focus will be made on the influence of social proximity measure through personal relationships to explain the emergencies of business relationships. We propose a case study of the local productive system Mecanic Vallée based on a mixt methodology which propose quantitative results of a binary logit model completed by qualitative case studies of firms.

Keywords: Proximities, business relationships, cluster, mix method

\section{RESUMEN}

Nuestra propuesta busca estudiar el impacto de diferentes formas de proximidad (geográfica, organizacional, cognitiva y social) para explicar la existencia de relaciones de negocios dentro de un clúster. Se presta especial atención a la influencia de la proximidad social, por medio de las relaciones personales, para explicar la formación de relaciones comerciales. Proponemos un estudio del Sistema Productivo Local "Mecanic Vallée", basado en un método mixto que propone a la vez resultados cuantitativos, a partir de un modelo logit binario, complementado con estudios de casos cualitativos.

Palabras Clave: Proximidad, relaciones de negocios, clúster, mixto método
$\mathrm{N}$ otre proposition cherche à comprendre comment les différentes formes de proximités expliquent l'émergence de relations d'affaires au sein d'un cluster. En effet, depuis la publication par M. Porter, en 1998, de l'article dans Harvard Business Review (Porter, 1998), de nombreux auteurs en économie se sont fortement intéressés aux éléments favorisant l'émergence ou le développement de clusters; notamment au rôle joué par les différentes formes de proximités. Que ces dernières soient spatiales ou non-spatiales, elles permettent de rapprocher les acteurs en mettant l'accent sur leurs points communs les incitant ainsi à agir ensemble et à innover (Torre et Rallet, 2005; Boschma, 2005). En revanche, le référentiel de la proximité a été moins utilisé pour expliquer l'émergence et l'existence de relations d'affaires, entendues comme des relations bilatérales et formalisées entre un client (le donneur d'ordres) et un fournisseur, un sous-traitant ou un cotraitant. Notre proposition cherche donc à comprendre comment les proximités expliquent l'émergence de relations d'affaires au sein d'un cluster industriel. Plus précisément, l'étude menée porte sur 153 adhérents du Système Productif Local (SPL) Mecanic Vallée (MV). Cette association loi 1901, localisée dans les départements français du Lot, de l'Aveyron et de la Corrèze fédère des entreprises, des collectivités, des acteurs de la formation pour les faire travailler ensemble autour de deux thématiques centrales : la métallurgie et la mécanique. Dans notre étude, la variable expliquée est l'existence de relations d'affaires recensées entre les membres du SPL. Les variables explicatives sont les différents types de proximités (géographique, organisationnelle, cognitive et sociale). Une attention particulière est portée à l'influence de la proximité sociale via les relations personnelles. L'étude est constituée d'un modèle logit complété par une analyse qualitative.

L'originalité de la recherche repose sur quatre points. La première originalité de notre travail porte sur la variable expliquée : les relations d'affaires entre firmes membres d'un cluster.

* Remerciements:

Nous remercions chaleureusement M. Hervé DANTON, M. Armando NEVES et M. Robert VITRAT membres de la Mecanic Vallée pour leur efficace soutien dans l'organisation de l'enquête de terrain. Nous remercions toutes les personnes contactées qui ont accepté de répondre à nos questions et d'échanger avec nous. Nous remercions également les rapporteurs anonymes qui nous ont aidés à améliorer ce travail, les erreurs demeurant dans cet article restant de notre entière responsabilité. 
D’autre part, ce travail se base sur une méthode mixte avec des résultats quantitatifs complétés par des résultats qualitatifs. La troisième originalité vient de la mesure de la proximité sociale par l'utilisation de plusieurs indicateurs permettant, notamment, de mesurer les relations personnelles. Enfin, le cluster étudié est situé sur un territoire constitué de villes moyennes en dehors des grandes agglomérations et des zones rurales.

Larticle est organisé en trois parties. La première effectue une revue de la littérature consacrée à la définition des concepts de proximité et de relations d'affaires ainsi qu'aux facteurs explicatifs des relations d'affaires. Dans une deuxième partie, c'est la construction des données et la méthode qui sont traitées. Les variables explicatives des relations d'affaires entre membres de la MV sont définies et les paramètres retenus pour tester chaque type de proximité sont présentés. Enfin, la troisième partie porte sur les résultats issus de plusieurs modèles testés mais aussi sur des éléments recueillis dans le cadre d'études de cas effectuées auprès de membres du cluster qui sont impliqués dans des relations d'affaires.

\section{Comment expliquer les relations d'affaires dans un cluster?}

L'objectif de notre étude est de comprendre quels facteurs peuvent expliquer les relations d'affaires dans un cluster. Pour mieux répondre à cet objectif, nous reviendrons dans un premier temps sur la définition des concepts de cluster et de SPL, puis nous évoquerons la grille d'analyse des formes de proximités. Nous définirons ensuite le concept de relations d'affaires, enfin nous reviendrons sur les travaux identifiés dans la littérature pour expliquer les relations d'affaires dans le contexte d'un cluster.

\section{SPL, DISTRICTS INDUSTRIELS ET CLUSTERS}

Notre étude de cas se situe dans le contexte d'un cluster. En effet, un cluster se définit comme "des concentrations géographiques d'entreprises et d'institutions reliées entre elles dans un champ d'activité spécifique» (Porter, 1998, p. 78). Nous nous appuyons ici sur la définition générale du concept proposé par Porter en 1998 qui a ensuite été largement popularisée et a donné lieu à un grand nombre de travaux et de politiques fondés sur ce concept (Vicente, 2017). En France, cette définition a inspiré la politique des pôles de compétitivité. Ce concept très général a été ensuite développé par Porter (2000) pour expliciter l'impact positif de ce phénomène de clustering sur la compétitivité d'un territoire ou d'une industrie. Cette définition très générale du cluster, qualifiée de «floue» par certains auteurs (Martin et Sunley, 2003), recouvre un très large panel de situations qui se fondent sur des compréhensions et des articulations différentes des trois éléments définissant les clusters : la concentration géographique, le champ commun d'activités et les relations existantes entre les acteurs.

Quand à eux, Les SPL, mis en place depuis 1997, se définissent comme : "une organisation productive concentrée sur un territoire correspondant à un bassin d'emploi qui fonctionne comme un réseau d'interdépendance, constitué d'unités productives de petite et moyenne taille, ayant des activités similaires ou complémentaires, qui se divisent le travail qui collaborent à moyen ou long terme» (Pommier, 2002). Ainsi, tout comme les pôles de compétitivité, les SPL sont des clusters dans le sens où ils reposent bien sur une concentration géographique d'acteurs liés entre eux (essentiellement des entreprises et majoritairement des PME), sur un territoire, dans un champ d'application donné (plutôt dans le domaine de la production ou autour d'un couple produit/marché) et reliées entre eux par l'existence de relations interentreprises fortes.

Ainsi les SPL respectent parfaitement les trois critères de définition d'un cluster. Toutefois, ces SPL se rapprochent d'une forme très spécifique des clusters, antérieure à eux : les districts industriels. En effet, la définition de ces SPL est basée sur la notion de districts italiens telle que construite par Becattini (1992) qui s'est lui-même inspiré du concept de district marshallien. Ce dernier regroupe dans une même localité des firmes spécialisées, impliquées dans la même industrie et qui bénéficient d'externalités d'agglomérations (Marshall, 1920).

La problématique de notre étude est d'autant plus pertinente qu'elle s'intéresse à un cluster de type district industriel et que dans ce dernier, les PME ont une place prédominante et les effets de localisation sont importants. Ainsi, le choix d'un SPL est donc plus pertinent que celui d'un pôle de compétitivité. En effet les pôles de compétitivité mobilisent une multiplicité d'acteurs (entreprises et organismes de recherche publique), dans des domaines variés (et pas uniquement industriels), dans des territoires plus étendus que les SPL (Guillaume, 2008). Nous allons pouvoir maintenant revenir sur les différentes formes de proximité que la littérature met en avant.

\section{QUELLE DÉFINITION DES PROXIMITÉS}

Les littératures, en économie, en gestion, mais aussi en sociologie ou en géographie mettent en avant une pluralité de typologies de proximités. Ces proximités peuvent faciliter tout type d'interactions, que ce soient des relations d'affaires ou des relations de coopérations pour l'innovation. En effet, la proximité signifie «être proche » et permet aux acteurs de bénéficier de points communs ou de ressources facilitant l'action. L'analyse en termes de proximités est structurée en trois courants principaux. Les premiers à s'intéresser à la question de la proximité sont des auteurs de l'École Française de la Proximité (Torre et Rallet, 2005; Carrincazeaux et al., 2008). Ces auteurs proposent de distinguer deux types de proximités : la proximité géographique et la proximité non-géographique (organisée). Par la suite, d'autres auteurs de cette même Ecole Française de la Proximité (Kirat et Lung, 1995; Talbot, 2008; Rychen et Zimmerman, 2008) proposent de distinguer trois formes de proximités : la proximité géographique, la proximité organisationnelle et la proximité institutionnelle. Enfin, Ron Boschma (2005) proposa une typologie autour de cinq formes de proximités que définirons par la suite.

Quels que soient les auteurs, la proximité géographique se définit par opposition à une distance. Elle est le plus souvent définie et mesurée de manière relative (Rallet et Torre, 2004). Cette proximité a pour effet de réduire les coûts, notamment les coûts de transactions et de transports, mais aussi de faciliter les interactions en face à face. La proximité géographique va pouvoir se mesurer soit à travers une distance kilométrique (Balland et al., 2013; Balland et al., 2016; Ledunois-Adam et al., 2010), soit par l'appartenance à une même unité géographique (région, département, unité urbaine), Bernela et Levy, 2016 ou Levy et Talbot, 2015 ont par exemple utilisé la localisation dans un même département. 
La proximité organisée représente «la capacité qu’offre qu’une organisation de faire faire interagir ses membres» (Rallet et Torre, 2004, p. 27). Cette proximité peut se définir par l'appartenance à une même organisation. Cette appartenance va faciliter les interactions entre ses membres en raison de l'existence de règles et routines communes à l'organisation et va multiplier les occasions d'interagir au sein de l'organisation. Mais elle peut aussi se définir entre organisations qui partagent des règles de fonctionnement communes.

À la suite de cette première distinction, certains auteurs de l'École Française de la Proximité (Kirat et Lung, 1995; Talbot, 2008; Rychen et Zimmerman, 2008) ont proposé de distinguer deux formes de proximités qui, ensemble, constituent la proximité organisée observée ci-dessus : la proximité organisationnelle et la proximité institutionnelle. Cette distinction s'ancre dans une perspective interactionniste par opposition à la distinction entre proximité organisée et géographique qui se situe plutôt dans une perspective institutionnaliste. Pour les institutionnalistes, les interactions vont automatiquement de pair avec les institutions : une interaction ne pouvant être pensée sans règles communes, alors que chez les interactionnistes, les interactions peuvent se passer des règles.

La proximité institutionnelle correspond au partage d'un même cadre institutionnel et donc au partage d'un ensemble de règles, de codes, de normes (Zimmermann, 2008). Par nature, cette forme de proximité introduit une dimension politique (Talbot, 2008). La proximité organisationnelle se définit quant à elle, comme une forme particulière de proximité institutionnelle entre acteurs ou organisations qui partagent un même espace social, par exemple une règle ou une coutume (Talbot, 2008).

Enfin, en adoptant également une vision interactionniste, Ron Boschma (2005) propose d'étendre les classifications précédentes autour de cinq formes de proximités. Il propose deux définitions plus restrictives des proximités organisationnelle et institutionnelle. La proximité organisationnelle se définit par le partage d'un même agencement organisationnel (à l'intérieur d'une organisation ou entre des organisations). Deux entités qui fonctionnent de la même façon pourront plus facilement partager des connaissances. Cette proximité se mesure le plus souvent par l'appartenance à une même forme organisationnelle (Bernela et Levy, 2016) ou par l'appartenance à un même groupe (Balland et al., 2013). Pour Boschma, la proximité institutionnelle est liée à l'existence d'un cadre institutionnel commun qui sera favorable aux interactions entre acteurs. En effet, les règles et les normes permettent de rendre prévisibles les comportements des acteurs. Au-delà des proximités géographique, organisationnelle, institutionnelle, la construction de deux formes supplémentaires de proximités permet d'éviter les confusions entre les termes.

La proximité cognitive (distincte de la proximité institutionnelle) correspond au partage d'un cadre cognitif, ou cadre de pensée commun. Cette proximité renforce l'échange de connaissances tacites entre des institutions. En développant une telle proximité, les acteurs peuvent améliorer leur capacité d'absorption (Cohen et Levinthal, 1990). La proximité cognitive va ainsi s'exercer entre deux entreprises du même secteur d'activité et est mesurée à partir de la typologie NACE (Balland et al., 2016) ou par la production de produits similaires (Balland et al., 2013).
Enfin, l'intérêt de la typologie de Boschma est de proposer une dernière forme de proximité permettant de mesurer les relations sociales des agents : la proximité sociale. Cette proximité se définit par le fait que les relations économiques entre acteurs sont insérées dans des relations sociales (Granovetter, 1985). La proximité sociale peut se comprendre comme une proximité entre organismes, mais le plus souvent cette proximité va passer par des relations sociales entre individus et intégrera donc une dimension personnelle.

Nous avons opté pour la typologie de Boschma. En effet, même si nous sommes d'accord avec l'importance de la distinction fondamentale entre les formes de proximité géographique, organisationnelle et institutionnelle pour décrire les interactions entre organisations dans un SPL, cette typologie nous permet d'opérationnaliser deux formes particulières de proximités qui semblent centrales dans la constitution de la MV : la proximité sociale et la proximité cognitive. La proximité sociale permet d'introduire les relations interpersonnelles pour expliquer les relations d'affaires et la proximité cognitive permet de mettre en avant les compétences spécifiques nécessaires à la constitution d'un SPL (Grossetti et al., 2006).

\section{DIFFÉRENTES FORMES DE RELATIONS D'AFFAIRES.}

Les relations d'affaires correspondent aux relations entre un client (aussi qualifié de donneur d'ordres) et un fournisseur, un sous-traitant ou un cotraitant. Trois formes de relations d'affaires peuvent être distinguées : les relations d'achats avec un fournisseur, les relations de sous-traitance qui intègrent une élaboration du cahier des charges par le donneur d'ordres et les relations de cotraitance qui sous-entendent une élaboration commune du cahier des charges entre le donneur d'ordres et le sous-traitant.

Malgré la diversité des formes de relations d'affaires, l'ensemble de ces relations se caractérise par l'existence d'une relation bilatérale entre le donneur d'ordres et le vendeur; ces relations d'affaires excluant donc les partenariats multilatéraux. Ce sont également, par définition, des relations inter-organisationnelles et formalisées. Ces relations se définissent aussi par l'existence d'une subordination du sous-traitant par rapport au client (Houssiaux, 1957 ou Chaillou, 1977) par opposition aux relations de jointventure ou d'alliance entre deux entreprises. Cependant, quelle que soit la forme des relations d'affaires (y compris la relation la plus simple, à savoir l'achat entre le fournisseur et le client), ces dernières constituent un élément essentiel dans la définition de la stratégie des entreprises (Calvi et al, 2010).

\section{QUELS FACTEURS EXPLICATIFS DES RELATIONS D'AFFAIRES?}

Comme nous pouvons le voir dans le tableau 1, les proximités sont mobilisées dans un grand nombre d'analyses en gestion mais aussi en économie pour expliquer les relations d'affaires.

Dans plusieurs études, précédemment identifiées, consacrées à la recherche des déterminants des relations d'affaires dans un cluster, la grille des cinq formes de proximités n'est pas clairement mobilisée. En revanche, toutes soulignent le rôle de la proximité sociale pour expliquer les relations d'affaires dans un cluster (Connell et Voola, 2013; Tunisini et al., 2011; 
Arnould et Mohr, 2005). On notera que dans ces études, le rôle de la proximité géographique n'est pas examiné. D’autres études vont, au contraire, prendre clairement en compte tant les proximités non spatiales (pas uniquement la proximité sociale) que les proximités spatiales (Keeble et Nachum, 2002; LedunoisAdam et al., 2010; Waxell et Malmberg, 2007; Balland et al., 2013). Enfin, plusieurs auteurs vont aussi chercher à expliquer la complémentarité entre les relations économiques (d'affaires) et les relations sociales dans les clusters (Balland et al., 2016; Watts et al., 2006; Ferriani et al., 2013).
Par la suite, nous allons tenter de mettre en avant la place des différentes formes de proximités dans un cluster localisé non pas dans une métropole mais dans une région organisée autour de plusieurs villes moyennes, en comparaison, par exemple, aux études de Balland et al. (2016) à Valence en Espagne, de Ferriani et al. (2013) à Bologne en Italie ou de Watts et al. (2006) dans la ville de Sheffield en Grande-Bretagne. En effet, comme nous allons le préciser, nous proposons une étude d'un SPL situé sur l'axe constitué par les villes de Figeac, Rodez et Brive en France.

\begin{tabular}{|c|c|c|c|c|c|}
\hline \multicolumn{6}{|c|}{ TABLEAU 1} \\
\hline Référence & $\begin{array}{l}\text { Domaine d'activité et } \\
\text { terrain mobilisé }\end{array}$ & $\begin{array}{l}\text { Type de proximité } \\
\text { sociale mobilisée }\end{array}$ & $\begin{array}{l}\text { Autres types de } \\
\text { proximités mobilisées }\end{array}$ & $\begin{array}{l}\text { Type de relations } \\
\text { d'affaires }\end{array}$ & Principaux résultats \\
\hline $\begin{array}{l}\text { Arnould et } \\
\text { Mohr, } 2005\end{array}$ & $\begin{array}{l}\text { Cluster des peaux et } \\
\text { cuirs, Niger }\end{array}$ & $\begin{array}{l}\text { Liens } \\
\text { interpersonnels }\end{array}$ & $\mathrm{NC}$ & $\begin{array}{l}\text { Relations de } \\
\text { sous-traitance }\end{array}$ & $\begin{array}{l}\text { Les liens au sein du cluster } \\
\text { fournissent un avantage dif- } \\
\text { férentiel au cluster }\end{array}$ \\
\hline $\begin{array}{l}\text { Balland } \\
\text { et al., } 2013\end{array}$ & $\begin{array}{l}\text { Industrie mondiale } \\
\text { du jeu vidéo }\end{array}$ & $\begin{array}{l}\text { Production } \\
\text { commune d'un } \\
\text { produit (jeu vidéo) }\end{array}$ & $\begin{array}{l}\text { Proxi géographique } \\
\text { Proxi. sociale } \\
\text { Proxi. institutionnelle } \\
\text { Proxi. cognitive } \\
\text { Proxi. Organisationnelle }\end{array}$ & $\begin{array}{l}\text { Relations } \\
\text { d'affaires }\end{array}$ & $\begin{array}{l}\text { L'ensemble des formes de } \\
\text { proximités influence la créa- } \\
\text { tion de liens (rôle plus faible } \\
\text { des proximités cognitive et } \\
\text { institutionnelle) }\end{array}$ \\
\hline $\begin{array}{l}\text { Balland } \\
\text { et al., } 2016\end{array}$ & $\begin{array}{l}\text { Cluster «Toy Valley» } \\
\text { (industrie du jouet), } \\
\text { (Valence) Espagne }\end{array}$ & $\mathrm{NC}$ & $\begin{array}{l}\text { Proxi geographique } \\
\text { Proxi. sociale } \\
\text { Proxi. institutionnelle } \\
\text { Proxi. cognitive } \\
\text { Proxi. Organisationnelle }\end{array}$ & $\begin{array}{l}\text { Opportunités } \\
\text { de relations } \\
\text { d'affaires }\end{array}$ & $\begin{array}{l}\text { Le statut des acteurs (exper- } \\
\text { tise, réputation, visibilitél } \\
\text { influence la structuration des } \\
\text { réseaux d'affaires. }\end{array}$ \\
\hline $\begin{array}{l}\text { Connell et } \\
\text { Voola, } 2013\end{array}$ & $\begin{array}{l}\text { Cluster de } \\
\text { l'ingénierie, } \\
\text { Australie }\end{array}$ & $\begin{array}{l}\text { Confiance entre } \\
\text { les membres du } \\
\text { cluster }\end{array}$ & NC & & $\begin{array}{l}\text { Les relations de confiance } \\
\text { influencent (favorisent) l'avan- } \\
\text { tage compétitif du cluster. }\end{array}$ \\
\hline $\begin{array}{l}\text { Ferriani } \\
\text { et al., } 2013\end{array}$ & $\begin{array}{l}\text { Cluster des } \\
\text { multimédias, } \\
\text { Bologne Italie }\end{array}$ & $\begin{array}{l}\text { Relations } \\
\text { personnelles }\end{array}$ & $\mathrm{NC}$ & $\begin{array}{l}\text { Relations de } \\
\text { sous-traitance }\end{array}$ & $\begin{array}{l}\text { Les liens sociaux sont deux } \\
\text { fois plus influents que les } \\
\text { liens économiques dans les } \\
\text { relations entre entreprises. }\end{array}$ \\
\hline $\begin{array}{l}\text { Keeble et } \\
\text { Nachum } \\
\text { (2002) }\end{array}$ & $\begin{array}{l}\text { Cluster de } \\
\text { l'ingénierie et } \\
\text { consultance, } \\
\text { Royaume-Uni }\end{array}$ & $\begin{array}{l}\text { Relations } \\
\text { personnelles }\end{array}$ & Proximité géographique & $\begin{array}{l}\text { Relations } \\
\text { fournisseurs- } \\
\text { clients }\end{array}$ & $\begin{array}{l}\text { Les relations d'affaires sont } \\
\text { expliquées par la proximité } \\
\text { géographique et sociale via } \\
\text { des relations personnelles } \\
\text { entre entrepreneurs. }\end{array}$ \\
\hline $\begin{array}{l}\text { Ledunois- } \\
\text { Adam } \\
\text { et al.,2010 }\end{array}$ & $\begin{array}{l}\text { Parc industriel } \\
\text { fournisseur, France }\end{array}$ & Capital social & $\begin{array}{l}\text { Proximité } \\
\text { géographique+ } \\
\text { proximité } \\
\text { organisationnelle }\end{array}$ & $\begin{array}{l}\text { Relations } \\
\text { fournisseurs- } \\
\text { clients }\end{array}$ & $\begin{array}{l}\text { Les proximités entre entre- } \\
\text { prises favorisent la création } \\
\text { d'un capital social entre leurs } \\
\text { salariés, capital qui favorise } \\
\text { les relations entre ces } \\
\text { mêmes entreprises }\end{array}$ \\
\hline $\begin{array}{l}\text { Tunisini } \\
\text { et al., } 2011\end{array}$ & $\begin{array}{l}\text { Cluster de la } \\
\text { mécanique, Italie }\end{array}$ & $\begin{array}{l}\text { Liens avec les } \\
\text { fournisseurs } \\
\text { locaux }\end{array}$ & NC & $\begin{array}{l}\text { Relations } \\
\text { fournisseurs- } \\
\text { clients }\end{array}$ & $\begin{array}{l}\text { Les PME multinationales } \\
\text { choisissent des fournisseurs } \\
\text { locaux pour leurs compé- } \\
\text { tences (innovation, réacti- } \\
\text { vité, performance). }\end{array}$ \\
\hline $\begin{array}{l}\text { Watts et al., } \\
2006\end{array}$ & $\begin{array}{l}\text { Cluster de la } \\
\text { métallurgie, } \\
\text { Sheffield, } \\
\text { Royaume-Uni }\end{array}$ & $\begin{array}{l}\text { Mobilité } \\
\text { professionnelle } \\
\text { du manager }\end{array}$ & NC & $\begin{array}{l}\text { Relations } \\
\text { fournisseurs- } \\
\text { clients }\end{array}$ & $\begin{array}{l}\text { L'encastrement des entre- } \\
\text { prises dans des liens locaux } \\
\text { d'affaires est influencé par } \\
\text { l'implication personnelle des } \\
\text { dirigeants d'entreprises dans } \\
\text { les réseaux. }\end{array}$ \\
\hline $\begin{array}{l}\text { Waxell et } \\
\text { Malmberg, } \\
2007\end{array}$ & $\begin{array}{l}\text { Cluster des } \\
\text { biotechnologies, } \\
\text { Suède }\end{array}$ & $\begin{array}{l}\text { Relations sociales } \\
\text { Mobilité du travail }\end{array}$ & Proximité géographique & $\begin{array}{l}\text { Relations } \\
\text { d'affaires }\end{array}$ & $\begin{array}{l}\text { Le cluster repose sur une } \\
\text { mobilité du travail et des } \\
\text { relations sociales à l'échelle } \\
\text { locale. Les marchés se si- } \\
\text { tuent à l'échelle globale. }\end{array}$ \\
\hline
\end{tabular}




\section{Données et méthode}

\section{LE SPL DE LA «MECANIC VALLÉE »}

La MV est un SPL situé dans le Sud-Ouest de la France (cf. carte 1) et spécialisé dans la métallurgie et la mécanique. Comme rappelé précédemment, les SPL sont des formes particulières de clusters, inspirées des districts industriels définis par Becattini (1992). Les SPL, tout comme les districts, s'inscrivent dans une histoire longue et dans une communauté locale. La compréhension de la structuration des relations d'affaires dans ce cluster ne peut se faire sans s'intéresser à sa trajectoire historique qui se décompose en trois étapes.

La première étape s'est déroulée avant 1998. Trois initiatives locales de développement étaient alors menées. D’une part, l’Union Européenne incite à la structuration de cellules locales de développement grâce à des appels à projets. Les départements du Lot et de l'Aveyron sont retenus sur des dossiers librement construits et basés sur le tissu économique alors existant. Le Lot cherche à structurer une filière mécanique et agroalimentaire et à combler son retard de développement économique. L'Aveyron, quant à lui, a déjà structuré une filière mécanique et métallurgique mais ce territoire cherche à se restructurer suite aux échecs de reconversion et d'implantation du site de Decazeville (Guillaume, 2005). Il souhaite aussi renforcer la coopération économique entre entreprises autour de technologies communes grâce, notamment, à l'implantation récente d'une école d'ingénieurs. L'objectif est alors de renforcer les partenariats entre industrie, formation et recherche (Haas, 2007). La Corrèze est, quant à elle, en train de structurer une filière mécanique mais, cette fois-ci, grâce aux Fonds de Restructuration des Entreprises de la Défense (FRED). Ces fonds sont versés aux zones d'emploi ayant fait l'objet d'une restructuration des entreprises de la défense. En Corrèze, il s'agissait d'effacer les nombreuses pertes d'emplois de l'usine GIAT de Tulle (aujourd'hui NEXTER). Pour limiter les conséquences sur l'emploi, l'État a cherché à dynamiser et coordonner l'ensemble des entreprises locales travaillant dans la mécanique et la métallurgie afin que les salariés licenciés puissent aisément retrouver du travail.

Une deuxième étape intervient en 1998. Les fonds européens sont épuisés. La France reprend l'initiative et lance les appels à projets pour les SPL. Les trois initiatives départementales de développement décident de s'unir et de déposer un dossier commun autour des spécialités productives communes que sont la métallurgie et de la mécanique. Ce dossier a été retenu et l'effort de structuration des filières a changé de forme et de nature. Il s'agissait de travailler sur trois départements différents en menant une action innovante. Le SPL s'est ainsi constitué en association en 2000 sous l'influence de 15 industriels fondateurs (5 par département). Les structures publiques n'étaient plus les porteurs de l'initiative mais des acteurs associés. Les industriels ont donc mené un certain nombre d'actions collectives dont la première fut, en 1999, les rencontres annuelles d'affaires. Les industriels ont aussi poursuivi les relations de formation, de recherche et d'industrie en coordonnant l'ensemble des parties prenantes (Haas, 2007). Parallèlement, la MV a essayé de développer ses marchés en se transformant de SPL à SLC en s'appuyant sur des sous-traitants de spécialité développant des compétences fondées sur des ressources cognitives (Grossetti et al., 2006). Ce SLC suit une trajectoire territoriale similaire à celle de l'industrie de la coutellerie à Châtellerault (Ferru, 2009) ou l'industrie des arômes à Grasse ou des chantiers navals de La Ciotat (Mendez et Mercier, 2006).

Une troisième étape de développement a été construite par les industriels à partir des années 1980. Il s'agit d'une politique ininterrompue d'essaimage (Guillaume, 2001 et 2005). Ces essaimages ont permis la constitution de nouvelles entreprises mais aussi un certain nombre de relations commerciales en raison des effets de sous-traitance créés. Cet essaimage a fait émerger les principaux donneurs d'ordres comme architectes de la recherche et de l'innovation dans le SPL (Guillaume, 2001).

Ainsi, entre 2012 et 2015, la MV a rassemblé 153 adhérents (entreprises et/ou établissements) ${ }^{1}$ essentiellement dans le secteur de la fabrication de produits métalliques (particulièrement les activités d'usinage, de tournage et de fraisage de pièces métalliques) mais aussi dans le domaine de l'ingénierie (cf. tableau 2). Ces entreprises sont essentiellement des PME ou TPE (plus de $85 \%$ de l'effectif d'entreprises), même si l'on retrouve aussi quelques grands groupes qui, pour certains, ont été des fondateurs de la MV. Enfin, en termes de localisation, ces établissements sont essentiellement répartis entre les trois départements fondateurs de la MV comme nous pouvons le voir dans la carte 1.

\section{CARTE 1}

\section{Localisation des 153 entreprises membres de la MV}

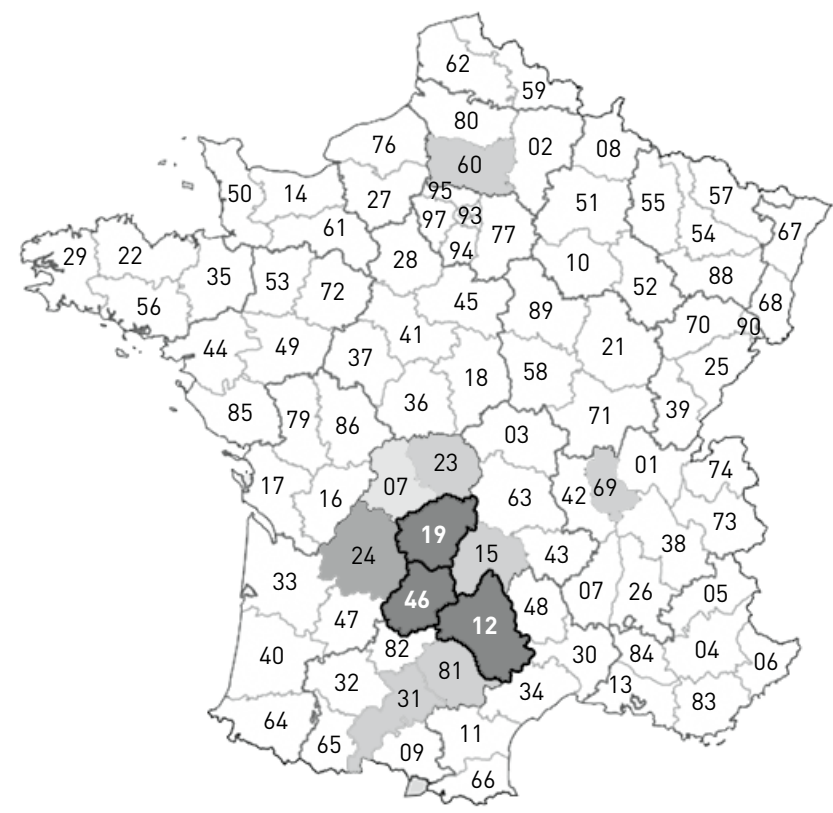

Légende : les numéros indiqués correspondent aux départements français. La couleur est proportionnelle aux nombre d'entreprises adhérentes à la MV entre 2012 et 2015. Les trois départements encadrés en noir correspondent aux trois départements fondateurs de la MV et accueillent chacun entre 35 et 45 adhérents.

1. Nous avons établi cette liste à partir des catalogues recensant de manière exhaustive les adhérents de l'Association en 2012, 2013 et 2015. Nous nous sommes concentrés sur la période 2012-2015 pour éliminer les anciens adhérents qui n’ont plus de liens directs avec la MV. 


\section{CONSTRUCTION DES DONNÉES SUR LES RELATIONS ENTRE MEMBRES DE LA MV}

Afin de pouvoir construire notre modèle, la première étape a été de recenser l'ensemble des 153 établissements ${ }^{2}$ adhérents de la MV, ou qui l'ont été au moins une fois entre 2012 et 2015 , et encore en activité en $2016^{3}$. Les données sur le secteur d'activité (code NAF au niveau 2), l'activité (plus détaillée selon la classification du site Kompass), la date de création, le statut juridique et la localisation géographique des établissements ont ensuite été identifiées à l'aide des données de l'INSEE et du site Kompass.

L'objectif de notre étude est d'expliquer la relation d'affaires entre deux entreprises. Ces relations d'affaires ont été repérées à partir d'une analyse du site internet et de la documentation de la Mecanic Vallée, ainsi que des sites internet des entreprises. Les données sur le type de relations d'affaires (soustraitance, cotraitance, ou relation clients-fournisseurs) étant confidentielles, notamment le rôle du donneur d'ordres dans le cahier des charges, nous proposons ici une définition élargie de la relation d'affaires englobant ces trois formes de relations. D'autre part, nous n'avons pas pu trouver l'information sur les relations d'affaires pour 67 firmes sur les 153 entreprises identifiées au préalable. Ainsi, le reste de l'analyse porte sur un échantillon de 86 firmes membres de la MV qui communiquent sur leurs relations d'affaires. Comme nous pouvons le voir dans le tableau 2, l'échantillon étudié de 86 entreprises est représentatif de l'ensemble des 153 entreprises adhérentes de la MV sur l'ensemble des variables caractéristiques des entreprises ${ }^{4}$.

Étant donné que notre étude porte sur l'étude des facteurs explicatifs d'une relation d'affaires entre deux entreprises, notre unité d'analyse ne sera pas l'entreprise membre de la MV, mais le lien entre deux entreprises membres de la MV. Nous proposons ainsi un modèle sur $86^{\star} 85 / 2$ soit 3655 liens possibles (les liens étant considérés comme symétriques entre deux entreprises). Ainsi, la variable explicative du modèle, $\mathrm{RA}=1$ si une relation d'affaires entre deux entreprises a été déclarée par l'une des deux entreprises en 2015, que cette relation ait eu lieu en 2015 ou antérieurement. Au total, 147 liens d'affaires entre membres ont été identifiés, soit $4.02 \%$ des liens possibles, et 61 entreprises sur $86(70 \%)$ déclarent avoir au moins une relation d'affaires avec un autre membre de la MV.

\section{MESURE DES FORMES DE PROXIMITÉ}

Nous avons proposé une mesure de la proximité géographique proxi_geo entre deux firmes, égale à 1 si les deux entreprises se situent dans la même aire urbaine. Nous avons choisi de nous focaliser sur une mesure fine de la proximité géographique dans l'aire urbaine, car la définition du territoire du cluster autour de trois départements est déjà un territoire peu étendu. En complément de la mesure de la proximité géographique, nous avons utilisé des variables de contrôle sur la localisation des entreprises dans les départements du Lot (variable $d p t 46$ ), de l'Aveyron ( $d p t 12)$ et de la Corrèze ( $d p t 19)$, à savoir les trois départements inclus dans les statuts de la MV pour vérifier l'existence d'une proximité géographique associée à une proximité institutionnelle au sein de chaque département.

Pour mesurer la proximité cognitive, nous avons utilisé la typologie des activités des firmes proposée par le site Kompass permettant une analyse plus fine des activités (cf. tableau 2 pour la liste des principales activités) et donc des ressources cognitives des entreprises étudiées. Plus précisément, nous avons défini une variable discrète proxi_cognitive variant de 0 à 7 et égale au nombre d'activités communes entre deux entreprises impliquées dans des relations d'affaires. Cette variable discrète mesure donc la similarité des activités de deux entreprises de la MV via le partage de blocs de compétences similaires (Cagli et al., 2009). En nous basant sur la classification en secteurs d'activités en 88 catégories (code NAF niveau 2 de l'INSEE), deux variables de contrôle ont été intégrées aux modèles permettant de mesurer l'impact de l'appartenance des entreprises aux secteurs d'activité les plus représentés au sein de la MV : la fabrication de produits métalliques (variable NAF25) et les activités d'ingénierie (variable NAF71).

Pour mesurer la proximité organisationnelle, nous avons pris en compte la forme organisationnelle de l'entreprise en nous basant sur la taille des effectifs et du statut (indépendant ou non) des entreprises (en adoptant une méthode assez similaire à celle de Levy et Talbot, 2015). Ainsi, nous distinguons 4 catégories de membres : les TPE indépendantes de moins de 10 salariés; les PME indépendantes de 10 à 500 salariés; les établissements de groupes (qualifiés de filiales) et enfin les établissements indépendants de plus de 500 salariés (ETI ou grands groupes). Nous avons opté pour une typologie assez simplifiée, mais qui permet de représenter les principales catégories d'aménagement organisationnel présentes dans la MV; même si cette typologie ne permet pas de prendre en compte l'ensemble des aménagements organisationnels identifiés par Mintzberg (1982). À partir de cette classification, nous avons construit la variable proxi_orga égale à 1 si les entreprises considérées ont la même forme organisationnelle. Des variables de contrôle sur chaque forme organisationnelle des entreprises ont été ajoutées (variables : TPE, PME et filiale).

Enfin, nous avons proposé trois mesures différentes de la proximité sociale. Dans un premier temps, nous avons mesuré la proximité sociale à travers les relations de mobilité professionnelle. Plus précisément, nous avons identifié les trajectoires professionnelles de l'ensemble des mandataires sociaux actuels des 86 entreprises de notre échantillon ${ }^{5}$ depuis 2004 et nous avons défini la variable proxi_sociale_mobilité. Cette dernière est égale à 1 si l'un des mandataires d'une des deux entreprises travaille ou a déjà travaillé dans l'autre entreprise en tant que mandataire.

2. Les données ont été identifiées et localisées au niveau de l'établissement adhérent à la MV et pas au niveau des entreprises. Plus précisément, en cas d'entreprises pluri-établissements (51 entreprises sur 154 au total), la firme a été localisée au niveau du siège social de l'entreprise si ce dernier se trouvait dans le territoire de la MV (4 cas sur 51). La firme a été localisée au niveau de l'établissement dont l'adresse était indiquée sur l'annuaire de la MV si ces coordonnées étaient disponibles (32 cas sur 51). Enfin pour les 14 cas restant, l'information a été cherchée sur le site internet des entreprises.

3. 11 entreprises adhérentes de l'association ont disparu entre leurs adhésion à la MV et 2016.

4. Un test du Khi2 a été réalisé pour confirmer la représentativité de l'ensemble de l'échantillon.

5. Les mandataires sociaux sont les personnes ayant autorité pour représenter la société auprès de tiers. Cette expression désigne les gérants, les directeurs généraux, les présidents directeurs généraux en priorité mais aussi les administrateurs par exemple. Les noms des 269 mandataires sociaux de ces 153 entreprises au 12 août 2016 ont été identifiés à partir des sites Societe.com, PointRisk et Orbis. Ensuite, les trajectoires professionnelles ont été identifiées via les sites societe.com et dirigeant.com. 


\begin{tabular}{|c|c|c|c|c|c|}
\hline & \multirow[b]{2}{*}{ Variables } & \multicolumn{2}{|c|}{$\begin{array}{l}\text { Entreprises membres de } \\
\text { la MV entre } 2012 \text { et } 2016\end{array}$} & \multicolumn{2}{|c|}{$\begin{array}{l}\text { Échantillon } \\
\text { d'entreprises étudiées }\end{array}$} \\
\hline & & Nb & $\%$ & $\mathbf{N b}$ & $\%$ \\
\hline \multirow{4}{*}{$\begin{array}{l}\text { Date de création } \\
\text { de l'entreprise }\end{array}$} & Avant 90 & 54 & $35 \%$ & 31 & $36 \%$ \\
\hline & Entre 90 et 99 & 39 & $25 \%$ & 20 & $23 \%$ \\
\hline & De 2000 à 2009 & 38 & $25 \%$ & 22 & $26 \%$ \\
\hline & De 2009 à 2016 & 22 & $14 \%$ & 13 & $15 \%$ \\
\hline \multirow{4}{*}{$\begin{array}{l}\text { Type } \\
\text { d'établissements }\end{array}$} & TPE & 45 & $29 \%$ & 22 & $26 \%$ \\
\hline & PME & 87 & $57 \%$ & 49 & $57 \%$ \\
\hline & Filiale & 13 & $8 \%$ & 8 & $9 \%$ \\
\hline & Grand groupe ou ETI & 8 & $5 \%$ & 7 & $8 \%$ \\
\hline \multirow{8}{*}{$\begin{array}{l}\text { Secteur d'activité } \\
\text { (NAF) }\end{array}$} & Fabrication de produits métalliques & 65 & $42 \%$ & 31 & $36 \%$ \\
\hline & Activités d’ingénierie & 18 & $12 \%$ & 15 & $17 \%$ \\
\hline & Fabrication de machines et équipements & 10 & $7 \%$ & 7 & $8 \%$ \\
\hline & Travaux de construction spécialisés & 8 & $5 \%$ & 4 & $5 \%$ \\
\hline & Métallurgie & 5 & $3 \%$ & 2 & $2 \%$ \\
\hline & Fabrication d'équipements électriques & 5 & $3 \%$ & 2 & $2 \%$ \\
\hline & Collecte, traitement et élimination des déchets & 5 & $3 \%$ & 3 & $3 \%$ \\
\hline & Autres & 37 & $24 \%$ & 23 & $27 \%$ \\
\hline \multirow{14}{*}{$\begin{array}{l}\text { Activités } \\
\text { principales } \\
\text { (KOMPASS) }\end{array}$} & Usinage des pièces métalliques & 38 & $25 \%$ & 18 & $21 \%$ \\
\hline & Tournage et fraisage des pièces métalliques & 37 & $24 \%$ & 19 & $22 \%$ \\
\hline & Rectification des pièces métalliques & 22 & $14 \%$ & 8 & $9 \%$ \\
\hline & Travaux de montage & 20 & $13 \%$ & 10 & $12 \%$ \\
\hline & Découpe des pièces métalliques & 18 & $12 \%$ & 6 & $7 \%$ \\
\hline & Conseil en ingénierie pour les constructions mécaniques & 17 & $11 \%$ & 14 & $16 \%$ \\
\hline & Perçage des pièces métalliques & 17 & $11 \%$ & 8 & $9 \%$ \\
\hline & Production de constructions mécaniques & 17 & $11 \%$ & 9 & $10 \%$ \\
\hline & Prototypage & 17 & $11 \%$ & 10 & $12 \%$ \\
\hline & Alésage des pièces métalliques & 15 & $10 \%$ & 9 & $10 \%$ \\
\hline & Conseil en ingénierie électrique & 14 & $9 \%$ & 11 & $13 \%$ \\
\hline & Conseil en ingénierie pour la chimie, la physique et la biologie & 14 & $9 \%$ & 11 & $13 \%$ \\
\hline & Conseil en ingénierie du bâtiment et des travaux publics & 13 & $8 \%$ & 10 & $12 \%$ \\
\hline & $\begin{array}{l}\text { Conseil en ingénierie pour les infrastructures de transport } \\
\text { et le trafic }\end{array}$ & 13 & $8 \%$ & 10 & $12 \%$ \\
\hline \multirow[t]{6}{*}{ Départements } & 46 & 44 & $29 \%$ & 33 & $38 \%$ \\
\hline & 19 & 35 & $23 \%$ & 19 & $22 \%$ \\
\hline & 12 & 44 & $29 \%$ & 18 & $21 \%$ \\
\hline & 87 & 14 & $9 \%$ & 6 & $7 \%$ \\
\hline & 24 & 5 & $3 \%$ & 2 & $2 \%$ \\
\hline & Autres & 11 & $7 \%$ & 8 & $9 \%$ \\
\hline $\begin{array}{l}\text { Membre Fondateur } \\
\text { de la MV }\end{array}$ & Oui & 9 & $6 \%$ & 7 & $8 \%$ \\
\hline \multirow{7}{*}{$\begin{array}{l}\text { Nombre de } \\
\text { participations } \\
\text { aux réunions } \\
\text { d'affaires de la MV } \\
\text { depuis } 2007\end{array}$} & 0 & 24 & $16 \%$ & 8 & $9 \%$ \\
\hline & 1 & 26 & $17 \%$ & 15 & $17 \%$ \\
\hline & 2 & 25 & $16 \%$ & 15 & $17 \%$ \\
\hline & 3 & 20 & $13 \%$ & 13 & $15 \%$ \\
\hline & 4 & 23 & $15 \%$ & 16 & $19 \%$ \\
\hline & 5 & 35 & $23 \%$ & 19 & $22 \%$ \\
\hline & Grand Total & 153 & & 86 & $100 \%$ \\
\hline
\end{tabular}

Note : Parmi les 45 TPE identifiées, nous incluons également deux entreprises de type holding dont une seule est intégrée dans l'échantillon d'entreprises étudiées Parmi les grands groupes et ETI, ce sont en majorité des établissements de taille intermédiaire. En effet, cette catégorie ne regroupe qu'un seul grand groupe de plus de 1000 salariés à savoir le groupe Ratier Figeac au cœur de la MV. Le secteur d'activité a été identifié à partir de la classification au niveau 2 de l'INSEE. 
En effet, comme l'a par exemple montré Del Vecchio (2010), les conseils d'administration sont des lieux de partage de proximité. La présence dans un même conseil d'administration d'un mandataire peut donc être utilisée comme un indicateur de proximité sociale. Nous avons également mesuré la proximité sociale via la participation commune des deux entreprises aux réunions annuelles d'affaires organisées par la MV entre 2007 et $2015^{6}$. Nous avons ensuite proposé une mesure d'une proximité sociale temporaire telle que définie par Bathelt et al. (2004) dans le cas des participations à des foires commerciales. Nous avons ainsi défini une variable discrète proxi_sociale_reunion variant entre 0 et 5 et mesurant le nombre de réunions annuelles d'affaires communes auxquelles ont participé les entreprises.

Enfin, nous proposons une troisième forme de mesure de la proximité sociale, permettant de mesurer plus finement les relations personnelles entre les représentants des entreprises, via une enquête réalisée auprès de l'ensemble des entreprises du SPL ayant déclaré des relations d'affaires avec d'autres membres de la MV. Au cours du mois d'octobre 2016, nous avons effectué des envois de questionnaires, suivis d'une relance en décembre 2016 par courriel auprès des 86 entreprises étudiées pour identifier les relations personnelles entre représentants. Chaque représentant devait sélectionner les entreprises de la MV avec lesquelles il entretenait des relations personnelles. 86 enquêtes ont été envoyées, nous avons reçu une réponse de la part de 22 entreprises soit $26 \%$ des entreprises concernées et $57 \%$ des liens 7 .

Afin d'expliquer les relations d'affaires, nous proposons de tester un modèle logit binaire. Ce modèle est adapté à notre question de recherche visant à expliquer une variable dépendante (la création d'une relation d'affaires) entre deux entreprises, en fonction de plusieurs variables explicatives indépendantes (les formes de proximités reliant les deux entreprises) tout en introduisant des variables de contrôle sur les caractéristiques des entreprises. Le tableau 3 permet de synthétiser la définition des variables utilisées dans le modèle.

Modèle $1: R A=$ proxi_cognitive+proxi_geo+proxi_orga + proxi_ sociale_mobilite+naf $25+n a f 71+T P E+P M E+$ filiale $+d p t 12+$ dep46+dpt19+constante

Afin de mieux comprendre la place de la proximité sociale sous ses différentes formes, nous proposons, dans les modèles 2 et 3, d'intégrer les différentes mesures de la proximité sociale, à savoir la proximité sociale temporaire via une participation commune à des réunions de la MV dans le modèle 2, puis les résultats de l'enquête sur les relations personnelles dans le modèle 3. Le tableau 2 présente la définition, les variables et les résultats des modèles 2 et 3 .

\section{UNE MÉTHODOLOGIE D'ANALYSE QUANTITATIVE MAIS AUSSI QUALITATIVE}

Cependant, les relations personnelles étant importantes pour décrire le modèle, un modèle économétrique ne suffit pas pour bien comprendre la logique qui se cache derrière les relations d'affaires. C'est pourquoi nous proposons de compléter notre analyse quantitative et le modèle logit par deux études de cas complémentaires : une étude de cas d'une entreprise $\mathrm{X}$ qui a confié plus de dix relations d'affaires à autant d'entreprises du SPL; ce qui l'a placé au cœur du SPL, et d'une seconde entreprise Y qui a obtenu 3 relations d'affaires auprès de trois adhérents de la MV et une seconde entreprise. Ces études de cas, que nous détaillerons par la suite, ont été réalisées à travers des entretiens semi-directifs ${ }^{8}$ ainsi que par une analyse de la presse spécialisée et une étude des sites internet de ces adhérents.

Ces deux entreprises ont chacune une position différente dans le réseau d'affaires du cluster. X, ETI familial, localisé à Figeac créé en 1989 suite à un essaimage et qui occupe une place de donneur d'ordres avec 10 relations d'affaires avec d'autres entreprises de la MV. Cette ETI bénéficie d'un fort dynamisme économique depuis sa création et a mené une politique très active de croissance externe au sein dudit réseau. Y créé en 2005, est la filiale d'une entreprise de la MV. Il s'agit d'une entreprise sous-traitante de spécialité en ce sens qu'elle est la seule entreprise du cluster à agir dans son domaine qui propose des prestations d'ingénierie particulières. Cette entreprise recense 3 relations d'affaires.

\section{Résultats}

\section{LE Rôle DE LA PROXIMITÉ GÉOGRAPHIQUE}

On peut noter que dans les résultats du modèle 1, la plupart des formes de proximités sont fortement significatives pour expliquer l'existence d'une relation d'affaires entre deux entreprises. Les résultats de l'impact de la proximité géographique sont renforcés dans les modèles 2 et 3 dans lesquels la proximité géographique est clairement significative. Ainsi, la proximité géographique va positivement influencer la probabilité de créer une relation d'affaires entre deux entreprises. Même si l'ensemble de la MV constitue un territoire de dimension limitée (200 kilomètres environ), la proximité géographique dans une même aire urbaine dans des petites villes ou des villes moyennes semble fortement influencer la probabilité de créer une relation d'affaires au sein de la MV. Ainsi, si la proximité géographique est importante, elle doit être considérée à un niveau territorial fin même si la MV a pour objectif de faire travailler tous les industriels intéressés ensemble, quelle que soit leur implantation en termes d'aire urbaine ou de département. En revanche, la localisation dans les trois départements de la MV ne semble pas influencer la probabilité d'obtenir un contrat, à l'exception du territoire de la Corrèze. L'implantation dans ce département, apparaît comme significativement négative, ce qui peut s'expliquer par le moins grand nombre d'entreprises membres de la MV localisées dans ce département. Ces résultats sur le rôle de la proximité géographique mesurée finement dans une aire urbaine sont renforcés par les résultats des études de cas qualitatives.

6. Ces rencontres d'affaires de la MV ont lieu tous les ans depuis 2000 et permettent les échanges entre les membres de l'association. L'objectif de ces rencontres est la création d'une interconnaissance des adhérents afin de constituer un réseau professionnel local.

7. Si l'une des entreprises concernées par un lien répondait à l'enquête, nous considérions que le lien existait (ou n'existait pas) en se basant sur la seule réponse. Si les deux entreprises répondaient différemment à l'enquête (1.6\% de l'ensemble des liens et $9.7 \%$ des liens avec 2 réponses), nous considérions que le lien personnel existait si au moins un des deux répondants considérait l'existence de ce lien (en nous basant sur la méthodologie similaire adoptée par Bernela et Levy, 2016).

8. Plus précisément un entretien a été réalisé avec les mandataires de l'entreprise Y en octobre 2016 Cet entretien est issu d'un projet de recherche plus large dans lequel nous avons effectué une vingtaine d'entretiens avec les entreprises membres de la MV. 


\begin{tabular}{|c|c|c|c|c|c|c|}
\hline \multicolumn{7}{|c|}{$\begin{array}{r}\text { TABLEAU } 3 \\
\text { Présentation des variables et résultats des modèles }\end{array}$} \\
\hline Variable & Signification & nb & $\%$ & $\begin{array}{l}\text { Effets marginaux } \\
\text { (st. Erreur) } \\
\text { du modèle } 1\end{array}$ & $\begin{array}{l}\text { Effets marginaux } \\
\text { [st. Erreur) } \\
\text { du modèle } 2\end{array}$ & $\begin{array}{l}\text { Effets marginaux } \\
\text { [st. Erreur] } \\
\text { du modèle } 3\end{array}$ \\
\hline \multirow{7}{*}{$\begin{array}{l}\text { Proxi- } \\
\text { Cognitive }\end{array}$} & \multirow{7}{*}{$\begin{array}{l}\text { Nombre d'activités communes } \\
\text { entre } 2 \text { entreprises de la MV }\end{array}$} & $1: 214$ & 5.85 & \multirow{7}{*}{$\begin{array}{c}0.21 \\
10.29)\end{array}$} & \multirow{7}{*}{$\begin{array}{c}0.12 \\
(0.29)\end{array}$} & \\
\hline & & $2: 114$ & 3.12 & & & \\
\hline & & $3: 37$ & 1.01 & & & \\
\hline & & $4: 26$ & 0.71 & & & \\
\hline & & $5: 7$ & 0.19 & & & \\
\hline & & $6: 52$ & 1.42 & & & \\
\hline & & $7: 7$ & 0.02 & & & \\
\hline Proxi_orga & $\begin{array}{l}=1, \text { si les } 2 \text { entreprises ont la } \\
\text { même forme organisationnelle }\end{array}$ & 1456 & 44.9 & $\begin{array}{c}-4.01 * * * \\
(0.86)\end{array}$ & $\begin{array}{l}-3.53 * * * \\
(0.85)\end{array}$ & $\begin{array}{l}-3.72 * * * \\
(0.85)\end{array}$ \\
\hline Proxi_geo & $\begin{array}{l}=1, \text { si les } 2 \text { entreprises sont } \\
\text { localisées dans la même aire } \\
\text { urbaine }\end{array}$ & 282 & 8.7 & $\begin{array}{l}5.53^{* * *} \\
(0.09)\end{array}$ & $\begin{array}{c}4.75^{* * *} \\
(0.88)\end{array}$ & $\begin{array}{l}3.88^{* * *} \\
(.089)\end{array}$ \\
\hline $\begin{array}{l}\text { Proxi__ } \\
\text { social_- } \\
\text { mobilite }\end{array}$ & $\begin{array}{l}=1, \text { si l'un des mandataires } \\
\text { d'une des deux entreprises est } \\
\text { ou a été mandataire dans l'autre } \\
\text { entreprise }\end{array}$ & 6 & 0.2 & $\begin{array}{l}8.47^{* *} \\
(3.55)\end{array}$ & $\begin{array}{l}8.06^{* *} \\
(3.55)\end{array}$ & $\begin{array}{l}7.09 * * \\
(3.57)\end{array}$ \\
\hline \multirow{5}{*}{$\begin{array}{l}\text { Proxi_- } \\
\text { sociale_- } \\
\text { reunion }\end{array}$} & \multirow{5}{*}{$\begin{array}{l}\text { = nombre de participations } \\
\text { communes aux rencontres } \\
\text { d'affaires de la MV de } 2007 \text { à } 2015 \text { : } \\
\text { 2007, 2009, 2011, } 2013 \text { et } 2015\end{array}$} & $0: 1069$ & 18.7 & \multirow[t]{5}{*}{ I } & \multirow{5}{*}{$\begin{array}{c}1.41 * * * \\
(0.28)\end{array}$} & \multirow{5}{*}{$\begin{array}{c}1.18 * * * \\
(0.28)\end{array}$} \\
\hline & & $1: 1026$ & 29.1 & & & \\
\hline & & $2: 938$ & 28.9 & & & \\
\hline & & $3: 432$ & 16.5 & & & \\
\hline & & $4: 190$ & 6.9 & & & \\
\hline $\begin{array}{l}\text { Proxi_- } \\
\text { perso }\end{array}$ & $\begin{array}{l}=1 \text { si les représentants des } \\
2 \text { entreprises déclarent une } \\
\text { relation personnelle dans le } \\
\text { cadre de l'enquête }\end{array}$ & 404 & 22.39 & I & I & $\begin{array}{c}3.42 * * * \\
(0.78)\end{array}$ \\
\hline TPE & $\begin{array}{l}=1 \mathrm{sil} \text { l'une des } 2 \text { entreprises est } \\
\text { une TPE }\end{array}$ & 1639 & 40.91 & $\begin{array}{c}-5.02 * * * \\
(0.97)\end{array}$ & $\begin{array}{c}-4.02 * * * \\
(0.96)\end{array}$ & $\begin{array}{c}-3.98^{* * *} \\
(0.95)\end{array}$ \\
\hline PME & $\begin{array}{l}=1 \text { si l'une des } 2 \text { entreprises est } \\
\text { une PME }\end{array}$ & 2989 & 87.75 & $\begin{array}{c}-4.62 * * * \\
(0.98)\end{array}$ & $\begin{array}{c}-4.32 * * * \\
(0.97)\end{array}$ & $\begin{array}{c}-4.62 * * * \\
(0.97)\end{array}$ \\
\hline Filiale & $\begin{array}{l}=1 \mathrm{sil} \text { l'une des } 2 \text { entreprises est } \\
\text { une filiale }\end{array}$ & 652 & 13.80 & $\begin{array}{c}-3.60 * * \\
(1.08)\end{array}$ & $\begin{array}{c}-3.17 * * \\
(1.06)\end{array}$ & $\begin{array}{c}-3.00 * * \\
(1.05)\end{array}$ \\
\hline Dpt 12 & $\begin{array}{l}=1 \text { si l'une des } 2 \text { entreprises est } \\
\text { localisée dans l'Aveyron (12) }\end{array}$ & 1377 & 34.47 & $\begin{array}{l}-0.76 \\
(0.83)\end{array}$ & $\begin{array}{l}-0.58 \\
(0.81)\end{array}$ & $\begin{array}{l}-0.82 \\
(0.81)\end{array}$ \\
\hline Dpt 46 & $\begin{array}{l}=1 \text { si l'une des } 2 \text { entreprises est } \\
\text { localisée dans le Lot (46) }\end{array}$ & 2277 & 62.23 & $\begin{array}{c}1.02 \\
(0.85) \\
\end{array}$ & $\begin{array}{l}-0.09 \\
10.87)\end{array}$ & $\begin{array}{l}-0.21 \\
(0.86) \\
\end{array}$ \\
\hline Dpt 19 & $\begin{array}{l}=1 \text { si l'une des } 2 \text { entreprises est } \\
\text { localisée en Corrèze (19) }\end{array}$ & 1444 & 43.46 & $\begin{array}{c}-2.26 * * \\
(0.86)\end{array}$ & $\begin{array}{c}-2.71 * * \\
(0.85)\end{array}$ & $\begin{array}{c}-2.92 * * \\
(0.86)\end{array}$ \\
\hline Naf25 & $\begin{array}{l}\text { = } 1 \text { si l'une des } 2 \text { entreprises est } \\
\text { dans la fabrication de produits } \\
\text { métalliques }\end{array}$ & 1485 & 61.69 & $\begin{array}{c}0.17 \\
(0.72)\end{array}$ & $\begin{array}{c}0.21 \\
(0.72)\end{array}$ & $\begin{array}{l}-0.09 \\
(0.72)\end{array}$ \\
\hline NAF71 & $\begin{array}{l}=1 \text { si l'une des } 2 \text { entreprises est } \\
\text { dans le secteur de l'ingénierie }\end{array}$ & 1170 & 35.97 & $\begin{array}{l}-1.07 \\
(0.76) \\
\end{array}$ & $\begin{array}{l}-1.02 \\
(0.75) \\
\end{array}$ & $\begin{array}{l}-1.15 \\
(0.74) \\
\end{array}$ \\
\hline & Total & 3655 & $100 \%$ & $\begin{array}{l}\text { LRChi2(12) } \\
=105.57\end{array}$ & $\begin{array}{l}\text { LRChi2(13) } \\
=133.35\end{array}$ & $\begin{array}{l}\text { LRchi2(14) } \\
=152.24\end{array}$ \\
\hline & PseudoR2 & & & 0.0856 & 0.1082 & 0.1235 \\
\hline
\end{tabular}

Source : données d'enquête. ${ }^{*} P<0.1 ; * * P<0.05 ; * * P<0.01$.

Dans le modèle 1, se situer dans la même aire urbaine qu'une autre entreprise augmente significativement (au seuil de $1 \%$ ) de $5,53 \%$ la probabilité d'une relation d'affaires avec cette entreprise

Les modèles 2 et 3 se définissent de la manière suivante :

Modèle 2: RA=proxi_cognitive+proxi_geo+proxi_orga+proxi_sociale_mobilite+proxi_sociale_reunion + naf25+naf71+TPE +PME+filiale+dpt12+dpt46+dpt19+constante Modèle 3: RA=proxi_cognitive+proxi_geo+proxi_orga+proxi_sociale_mobilite+proxi_sociale_reunion +proxi_perso+naf25+naf71+TPE +PME+filiale+dpt12+ dpt46+dpt19+ constante 
Pour la première étude de cas, on note que la politique de croissance externe de l'entreprise $\mathrm{X}$ se fait avec des entreprises à proximité géographique de son siège social. Au milieu des années 1990, cette entreprise a ainsi acquis deux sociétés toutes deux situées à proximité géographique (soit entre 20 et $30 \mathrm{~km}$ pour les deux entreprises) du siège de X. En 2004, X a participé à la création d'une entreprise localisée à 40 kilomètres de son siège, dans le Lot, puis a acquis deux autres entreprises en 2014; entreprises respectivement localisées à $43 \mathrm{~km}$ et à $1 \mathrm{~km}$ du siège. Ces rachats, bien que limités au cas de l'entreprise $\mathrm{X}$, soulignent aussi l'importance majeure de la proximité géographique (au sein même du territoire de la MV) pour développer les coopérations et les relations d'affaires. D'ailleurs il semble que ce rôle de la proximité géographique constitue une spécificité de ce SPL. En effet, Guillaume (2005) a proposé une comparaison des stratégies diversifiées des six principaux donneurs d'ordres du territoire dans le domaine automobile, de la machine-outil et de l'aéronautique. Cette étude montre que les entreprises automobiles sont faiblement rattachées au territoire contrairement à Ratier dans le secteur de la fabrication de modules pour l'industrie aéronautique qui a clairement développé une stratégie locale d'essaimage.

Le cas de l'entreprise Y confirme aussi le rôle de la proximité géographique (définie au sens strict de l'aire urbaine) puisqu'après sa création par une autre entreprise $(Z)$, l'entreprise Y a implanté ses deux bureaux d'études à Toulouse et à Figeac. Ce dernier est situé à quelques mètres du siège de $\mathrm{Z}$ et à moins d'un km de l'entreprise Ratier Figeac au cœur de la Mecanic Vallée. L'objectif de ces deux implantations (Toulouse et Figeac) est d'être proche et donc réactif tant auprès des donneurs d'ordres toulousains que lotois; notamment les entreprises $\mathrm{Z}$ et Ratier Figeac. Les autres entreprises en lien avec l'entreprise Y sont d'ailleurs toutes à proximité géographique les unes des autres. Cette entreprise souligne que : "Il est nécessaire de se rencontrer physiquement de manière régulière [...]. Avec tous les moyens de communication dont on dispose, il n'est pas moins important de se rencontrer physiquement. ". Ainsi, pour l'entreprise Y la proximité géographique est intimement liée à la possibilité de construire et maintenir une proximité sociale et une relation d'affaires.

\section{LE RÔLE DE LA PROXIMITÉ ORGANISATIONNELLE}

Dans l'ensemble des modèles testés, la proximité organisationnelle entre deux firmes est aussi une variable significative mais négative. Cela s'explique par le fait que dans la MV, les principaux donneurs d'ordres sont plutôt des ETI ou des grandes entreprises alors que les sous-traitants réalisant les travaux sont plutôt des petites entreprises (Guillaume, 2005). Ce résultat se confirme en notant que les variables de contrôle sur les formes organisationnelles d'entreprises de petite taille (TPE, PME et filiales) sont significatives (et négatives) par rapport à la variable de contrôle sur les grands groupes.

Bien que limitées aux cas de deux entreprises, nos études de cas renforcent les résultats du modèle logit. X joue essentiellement sur son statut d'ETI pour construire son rôle de donneur d'ordres dominant un important réseau principalement de PME agissant en mécanique générale, secteur le plus représenté dans le SPL. Dans le cas de l'entreprise Y, sous-traitant de spécialité inséré dans un réseau, c'est justement la petite taille, la proximité au client et la réactivité due à la petitesse de la structure qui vont être mises en avant pour se mettre au service des donneurs d'ordres locaux.

\section{LE Rôle de LA PROXIMITÉ COGNITIVE}

La proximité cognitive définie par le nombre d'activités communes entre 2 entreprises ne semble, en revanche, pas significative quel que soit le modèle testé. Ainsi, dans un même domaine industriel de la mécanique et de la métallurgie au sens large, si deux entreprises partagent un grand nombre d'activités, cette proximité cognitive, ne va pas favoriser ou au contraire diminuer la probabilité de relations d'affaires entre deux firmes. On peut aussi noter que le secteur d'activités de la fabrication d'objets métallurgiques et le secteur de l'ingénierie ne sont pas non plus significatifs pour expliquer la probabilité d'obtenir une relation d'affaires pour une firme. L'ensemble des secteurs d'activités de la MV est ainsi nécessaire à la constitution du SPL et les donneurs d'ordres vont faire leurs choix au sein du portefeuille local constitué d'activités variées. Cependant, ce résultat doit être relativisé. En effet, si notre indicateur permet de mesurer partiellement une proximité cognitive entre les membres du SPL, il ne permet cependant pas de mesurer finement le rôle des compétences spécifiques, notamment les compétences des entreprises, que Guillaume (2005), qualifient des soustraitants de spécialité. Cette mesure de la proximité cognitive par les compétences permettrait de mieux identifier le rôle de cette forme de proximité dans le SPL. Pourtant, comme l'ont souligné Grossetti et al. (2006) ces compétences sont au cœur du développement du système local de compétences de la MV.

Les études de cas permettent d'illustrer le rôle de la proximité cognitive sur des compétences spécifiques. Le groupe $\mathrm{X}$ s'est constitué autour de la mécanique générale et plus particulièrement de l'usinage, son cœur de métier. L'entreprise a acheté, progressivement, plusieurs PME plus petites qu'elle. Ces PME avaient pour particularité de travailler dans l'usinage mais aussi sur des niches spécifiques : usinage de petite dimension, fabrication de prototypes, usinage de pièces de grandes dimensions ou encore usinage et traitement de surface. Tous les achats étaient réalisés afin de maîtriser le plus de niches du cœur de métier de l'entreprise. Parmi ces achats existaient d'anciens sous-traitants de X qui ont été intégrés dans le but d'augmenter les capacités de production d'une entreprise à la croissance quasiment ininterrompue depuis sa création. En 2004, X a intégré une société d'ingénierie afin de disposer de l'ensemble des compétences nécessaires à la production de sous-ensembles. Cette société est un des fournisseurs de X.

$\mathrm{X}$ fait également appel à cinq usineurs indépendants qu'elle a choisi de ne pas racheter. En effet, ces cinq entreprises spécialistes de l'usinage sont des PME familiales implantées historiquement et qui permettent à $\mathrm{X}$ de pouvoir reporter sur elles la charge de travail de manière souple et réactive. De la même manière, $X$ va sous-traiter les activités d'entretien des machines et d'équipements électriques à plusieurs (quatre au total) soustraitants membres du SPL en favorisant, là aussi, les relations de proximité géographique et l'appartenance des sous-traitants à la MV. La diversification du portefeuille de compétences semble très importante dans cette stratégie. 
Le cas de l'entreprise Y s'explique par le besoin de complémentarité cognitive. Ainsi, si l'entreprise Y a été rachetée par l'entreprise Z, c'est parce que, en 2005, pour développer son entreprise et notamment la phase de conception et de R\&D, le fondateur de $\mathrm{Z}$ a décidé de créer une TPE : l'entreprise Y. Suite à cette création, l'entreprise va donc dissocier ses activités : l'entreprise $Z$ se spécialisera dans la fabrication. L'entreprise $Y$, quant à elle, se spécialisera dans la conception et l'ingénierie en regroupant l'ensemble de la $R \& D$ du groupe créé. Y a donc sa maison mère comme principal client. De plus, $\mathrm{Y}$ peut, par sa spécialité, intervenir auprès de nombreuses entreprises exerçant différentes spécialités, notamment celles ayant une forte activité dans l'ingénierie.

\section{LES DIFFÉRENTES FORMES DE PROXIMITÉ SOCIALE}

Enfin, notre analyse montre que la proximité sociale mesurée par la mobilité professionnelle des mandataires est positivement significative pour expliquer la probabilité d'une relation d'affaires entre deux firmes. Ce résultat provient de l'intégration, au sein de groupes, de sous-traitants après le rachat par le donneur d'ordres.

Concernant l'étude de cas de l'entreprise X, on note que la politique de croissance externe de $\mathrm{X}$ se traduit par une mobilité professionnelle des mandataires de cette entreprise. Ces mandataires unifient les stratégies des membres du groupe et créent une culture commune en même temps qu'ils renforcent les liens entre acquéreurs et anciens sous-traitants.

Le cas de l'entreprise Y est révélateur de l'importance des mobilités professionnelles des dirigeants pour obtenir des relations d'affaires. L'intégration de $\mathrm{Y}$ dans un réseau d'affaires au sein du cluster est fortement dépendante de l'histoire personnelle de son fondateur. Ce dernier a été cadre de Ratier-Figeac jusqu'en 1990. Cette année-là, ce même cadre est poussé par sa hiérarchie à créer l'entreprise $\mathrm{Z}$, à laquelle Ratier confiera des tâches de sous-traitance. Cette sous-traitance est fortement dépendante de la personnalité du fondateur qui inspirait suffisamment confiance à sa hiérarchie pour que celle-ci voit en lui un entrepreneur fiable en termes de production et capable d'assumer les responsabilités de gestionnaire. La relation d'affaires s'est maintenue dans le temps et Ratier reste le premier client de Z. En 2005, le fondateur de $Z$ décide de créer la société Y afin de disposer d'un bureau d'études et en devient immédiatement le gérant. La création de la relation d'affaires entre $\mathrm{Y}$ et $\mathrm{Z}$ a été associée au parcours professionnel du dirigeant. Enfin, en 2012, ce dirigeant prend sa retraite et revend son entreprise à trois des cadres de $\mathrm{Z}$ et $\mathrm{Y}$ qui se partagent les postes de direction des entreprises $\mathrm{Y}$ et $\mathrm{Z}$.

Dans le modèle 2, nous introduisons une mesure de la proximité sociale temporaire par la participation commune des entreprises aux rencontres d'affaires de la MV. Les résultats du modèle 2 ne diffèrent pas de ceux du modèle 1 . Dans le modèle 2, les deux formes de mesure de la proximité sociale semblent jouer un rôle dans le modèle, même si la participation aux réunions d'affaires de la MV semble plus fortement significative. Ainsi, ce résultat renforce l'importance du rôle de médiateur joué par la MV pour permettre la rencontre entre deux entreprises; un rôle de médiateur facilité par la proximité géographique entre les membres de la MV.
Le rôle des rencontres d'affaires est fréquemment souligné par les parties prenantes du SPL pour dynamiser leurs relations dans un cadre local et ainsi accéder à l'information pertinente pour créer des relations d'affaires. Ainsi, dans un entretien au journal local, l'animateur de ces rencontres précise que «le but de cette journée est de réunir tout le monde en un même lieu, pour faire fonctionner ce réseau de proximité, et favoriser une dynamique industrielle entre nous " (La Dépêche du Midi, 29 septembre 2015). De même, un dirigeant d'une PME à la fois donneur d'ordres et sous-traitante précise au journal la Montagne, le 27 septembre 2000 que : «on ne vient pas seulement voir les donneurs d'ordres. C'est un moyen d'échanger avec eux en marge de discussions purement commerciales. Il s'agit également de maintenir les liens avec nos sous-traitants locaux».

En dehors du critère de proximité géographique et de complémentarité cognitive qui semblent être les deux facteurs expliquant le choix des relations d'affaires de l'entreprise X, on peut aussi noter que l'ensemble des entreprises sous-traitantes de $\mathrm{X}$ a, au moins, croisé une fois $\mathrm{X}$ lors des rencontres annuelles de la MV. Notre étude de cas permet ainsi de souligner le rôle de médiateur joué par les réunions annuelles de la MV pour faciliter les relations d'affaires. Le mandataire de l'entreprise Y précise d'ailleurs : «on y va pour rencontrer des personnes et créer des liens avec d'autres entreprises, présenter nos savoir-faire et faire du relationnel ». La présentation des savoir-faire à ces réunions est ainsi considérée comme un préalable inconditionnel pour pouvoir rencontrer des interlocuteurs pertinents.

Enfin, dans le modèle 3, nous intégrons une troisième forme de proximité sociale, via la connaissance personnelle (le contact dans le répertoire téléphonique) de représentants d'entreprises de la MV. Il semble, au vu des résultats du $3^{\text {ème }}$ modèle, que la proximité sociale via des relations personnelles soit très fortement significative. Cette mesure de la proximité sociale via les relations personnelles a réduit la significativité de toutes les autres formes de proximités ce qui amène à conclure que le plus important dans la création d'une relation d'affaires est l'interconnaissance des individus qui peuvent l'initier, la formaliser et la conclure. Ce rôle des relations interpersonnelles confirme les résultats de Garzoun et Haddad (2016) qui ont souligné l'importance des réseaux personnels pour créer des opportunités d'affaires chez les entrepreneurs tunisiens, sans toutefois prendre en compte le rôle des autres formes de proximités. Les réunions d'affaires restent significatives car elles sont complémentaires à ces relations personnelles.

Les études de cas réalisées confirment aussi les résultats du modèle. Selon l'entreprise $\mathrm{Y}:$ «Ces réunions permettent de créer des contacts, de gagner de petits volumes d'affaires, d'être testés et de monter en puissance». Ces réunions permettent aussi d'entretenir les relations personnelles : "à l'heure actuelle, par le biais des rencontres de la $M V$, on reprend contact avec $X$ après que certains de ses salariés, qui étaient nos contacts, soient partis à la retraite». L'accès à l'information entre entreprises, notamment dans le cadre des rencontres annuelles d'affaires, passe par des contacts interindividuels. Toutefois, ces relations interpersonnelles sont jugées centrales dans le processus d'accès à l'information. Ainsi l'entreprise Y souligne l'importance des relations personnelles pour créer des relations d'affaires : «Ça ne marche pas d'aller taper à la porte. Cela ne porte pas ses fruits. 
Il est nécessaire d'avoir des relations, des recommandations. Il est important d'avoir un visage sur la société [...] pour obtenir ce que l'on va appeler un tuyau.» (Entretien téléphonique, janvier 2017). En revanche, pour toutes ces entreprises, l'interconnaissance personnelle ne signifie pas que les relations privées et les relations professionnelles se superposent.

\section{Conclusion}

L'objectif de cet article était de comprendre le rôle des différents types de proximités dans la création de relations d'affaires au sein d'un cluster industriel. L'article a démontré que la plupart des proximités étudiées a une influence significative dans la création de relations d'affaires et particulièrement les proximités géographique et sociale. La proximité cognitive au contraire paraît peu significative et c'est la différence entre les formes organisationnelles qui va favoriser l'émergence de relations d'affaires. Enfin la proximité sociale apparaît comme très fortement significative, quelle que soit la mesure adoptée. Notre travail permet de donc de mettre en avant le rôle important des relations de proximité sociale pour favoriser les relations d'affaires dans un contexte territorial. Cette étude permet aussi de souligner la complémentarité entre ces relations et les autres formes de proximités. Les proximités sociale et géographique se complètent pour favoriser les relations d'affaires entre firmes. De plus, cette conclusion est le résultat d'une étude fondée sur une méthodologie mixte basée à la fois sur une étude logit et sur des études de cas qualitatives.

Ces résultats amènent donc à préconiser la poursuite et l'accentuation des rencontres entre firmes menées par la MV. Ces résultats encouragent les instances de gouvernance des clusters ou des territoires à mettre en place des évènements permettant des rencontres entre leurs membres. Ces rencontres entre firmes sont aussi des rencontres entre individus. L'intensification de la fréquence de ces rencontres et leur enrichissement en termes de contenus constituent un axe majeur de la continuation de la politique de constitution d'un réseau d'affaires. Elle serait bénéfique à une logique territoriale, celle de la coopération entre membres pour une diversité de raisons, mais aussi bénéfique pour les membres du SPL qui voient leur santé économique renforcée. Certains membres du SPL sont aussi adhérents des Pôles de Compétitivité Aerospace Valley, Elopsys ou ViaMéca. Il serait intéressant de savoir si une telle proximité entre les adhérents à la fois du SPL et d'un pôle se poursuit. Ce maintien permettrait de faire perdurer la dynamique territoriale en œuvre mais il permettrait aussi aux membres du SPL d'enrichir les productions aux contenus technologiques. Cet enrichissement serait alors source d'une valeur ajoutée plus forte.

Ces résultats nous conduisent également à proposer des recommandations en direction des entrepreneurs en les encourageants à la fois à participer aux actions de gouvernance locales mis en place sur leurs territoires, mais aussi à favoriser le développement de relations sociales permettant de développer leurs portefeuilles de relations d'affaires.

Les limites de l'étude reposent sur quatre aspects. D’une part, les effets de lock-in ne sont pas étudiés. Boschma rappelle qu'une trop forte proximité entre firmes peut engendrer des effets de mimétisme et de trop grande confiance qui annuleraient les capacités d'adaptation et d'innovation du réseau (Boschma et al., 2002). De plus, l'étude devrait s'orienter vers des entretiens complémentaires pour mieux identifier et comprendre les cadres de la construction des relations interpersonnelles, mais également les mécanismes plus précis d'émergence et de maintien des relations d'affaires. Troisièmement l'effet de la proximité cognitive reste limité car il ne prend pas en compte les compétences très spécifiques des entreprises du SPL et, là aussi, des entretiens complémentaires permettraient de mieux analyser ces compétences. Enfin, l'impact des relations d'affaires sur la croissance et l'innovation des firmes n'est pas étudié et pourrait constituer une étape supplémentaire à ce travail.

\section{Bibliographie}

Arnould, Eric J.; MoHr, Jakki J. (2005). «Dynamic transformations for base-of-the-pyramid market clusters", Journal of the Academy of Marketing Science, Vol. 33, N³, p. 254-274.

Balland, Pierre-Alexandre; Belso-Martinez, José-Antonio; Morrison, Andrea (2016). «The dynamics of technical and business knowledge networks in industrial clusters embeddedness, status or proximity?», Economic Geography, Vol. 92, $\mathrm{N}^{\circ} 1$, p. 35-60.

Balland, Pierre-Alexandre; De VaAn, Mathijs; Boschma, Ron (2013). «The dynamics of interfirm networks along the industry life cycle : the case of the global video game industry 1987-2007 ", Journal of Economic Geography, Vol. 13, N5, p. 741-765.

Bathelt, Harald; Malmberg, Anders; Maskell, Peter (2004). "Clusters and knowledge : local buzz, global pipelines and the process of knowledge creation », Progress in human geography, Vol. 28, N 1, p. 31-56.

Becattini, Giacomo (1992). «Le district marshallien : une notion socio-économique», dans G. Benko et A. Lipietz (sous la direction de), Les régions qui gagnent, PUF, p. 37-39.

Bernela, Bastien; Levy, Rachel (2016). «Modalités de coordination de projets collaboratifs pour l'innovation : entre interactions en face-à-face et interactions à distance», Revue d'Économie Régionale \& Urbaine, N²016/2, p. 298-324.

Boschma, Ron (2005). «Proximity and innovation : a critical assessement», Regional studies, Vol. 39, N 1, p. 61-74.

Boschma, Ron; Lambooy, Jan; Schutjens, Veronique (2002). «Embeddedness and innovation», dans M. Taylor et S. Leonard (sous la direction de), Embedded entreprise and social capital : international perspectives, Ashagate, p. 19-35.

Cagli, Ayshe; Kechidi, Med; Levy, Rachel (2009). «Gestion stratégique de la supply-chain et firme-pivot dans le secteur aéronautique ", Revue française de gestion industrielle, Vol. 28, $\mathrm{N}^{\circ} 2$, p. 124-139.

Calvi, Richard; Paché, Gilles; Jarniat, Pierre (2010). «Lorsque la fonction achats devient stratégique», Revue Française de Gestion, Vol. 2010/6, N²05, p. 119-138.

Carrincazeaux, Christophe; Lung, Yannick; Vicente, Jérôme (2008). «The scientific trajectory of The French school of proximity : interaction and institutional-based approaches to regional innovation systems », European Planning Studies, Vol. 16, N 5, p. 617-628.

Chaillou, Bernard (1977). «Définition et typologie de la soustraitance», Revue économique, Vol. 28, N² 2, p. 262-285. 
Cohen, Wesley M.; Levinthal, Daniel A. (1990). «Absorptive capacity : a new perspective on learning and innovation", Administrative Journal Quarterly, Vol. 35, $\mathrm{N}^{\circ} 1$, p. 128-152.

Connell, Julia; Voola, Ranjit (2013). «Knowledge interaction and competitiveness : a longitudinal study of an industry cluster", Journal of Knowledge Management, Vol. 17, N², p. 208-225.

Del Vecchio, Nathalie (2010). «Réseaux de conseils d'administration et adoption de pratiques de gouvernance d'entreprise», Revue Française de Gestion, Vol. 36, №202, p. 145-161.

Ferriani, Simone; Fonti, Fabio; Corrado, Raffaelle (2013). «The social and economic bases of network multiplexity : exploring the emergence of multiplex ties », Strategic Organization, Vol. 11, $\mathrm{N}^{\circ} 1$, p. 7-34.

FERRU, Marie (2009). "Le développement territorial du Châtelleraudais: spécialisation cognitive et diversité des échelles spatiales", Géographie, Économie, Société, Vol. 11, N³, p. 193-212.

Garzoun, Lofti; HADDAD, Samia (2016). «Rôle du réseau personnel dans l'identification et l'exploitation d'opportunités d'affaires», Management International, Vol. 21, No 1, p. 58-71.

GranOVETTER, Mark (1985). «Economic action and social structure : the problem of embeddedness", American journal of sociology, Vol. 91, N³, p. 481-510.

Grossetti, Michel; Zuliani, Jean-Marc; Guillaume, Régis (2006). "La spécialisation cognitive: les systèmes locaux de compétences", Les Annales de la Recherche Urbaine, Plan Urbanisme-Construction - Architecture, No 101, p. 23-31.

Guillaume, Régis (2001). «Systèmes productifs localisés, action publique et innovation : l'exemple de la "Mecanic Vallée» ", Canadian Journal of Regional Science, Vol. 24, N 1, p. 121-140.

Guillaume, Régis (2005). «Les relations inter-entreprises dans la Mecanic Vallée», dans R. Guillaume (sous la direction de), Globalisation, systèmes productifs et dynamiques territoriales: regards croisés au Québec et dans le Sud-Ouest Français, L'Harmattan, p. 237-272.

HaAs, Joachim (2007). «Formation emploi Mecanic Vallée : Interactions entre système productiflocal et formation», Formation Emploi, $\mathrm{N}^{\circ}$ 97, p. 9-21.

Houssiaux, Jacques (1957). «Le concept de "quasi-intégration" et le rôle des sous-traitants dans l'industrie», Revue économique, Vol. 8, $\mathrm{N}^{\circ} 2$, p. 221-247.

KeEble, David; NAChum, Lilach (2002). «Why do business services firms cluster? Small consultancies, clustering and decentralization in London and Southern England», Transactions of the Institute of British Geographers, Vol. 27, N 1, p. 67-90.

KIRAT, Thierry; LunG, Yannick (1995). »Innovations et proximités : le territoire, lieu de déploiement des ressources d'apprentissage", dans N. Lazaric et j-M. Monnier (sous la coordination de), Coordination économique et apprentissage des firmes, Economica, p. 206-227.

Ledunois-Adam, Sonia; Baudasse, Thierry; Renault, Sophie (2010). «Proximité spatiale et capital social : le cas des Parcs Industriels et Fournisseurs", Management \& Avenir, Vol. 33, $\mathrm{N}^{\circ} 2010 / 3$, p. $14-34$.
LeVy, Rachel; TALвот, Damien (2015). «Control by proximity evidence from the "Aerospace Valley" competitiveness cluster", Regional studies, Vol. 49, N 6, p. 955-972.

Marshall, Alfred (1920). Principles of economics, Paris, V. Giard \& E. Brière, $661 \mathrm{p}$.

Martin, Ron; Sunley, Peter (2003). «Deconstructing clusters : chaotic concept or policy panacea?», Journal of Economic Geography, Vol. 3, N 1, p. 5-35.

Mendez, Ariel; Mercier, Delphine (2006). «Compétences-clés de territoires : le rôle des relations inter organisationnelles», Revue française de gestion, Vol. 164, N²006/5, p. 253-275.

Mintzberg, Henry (1982). Structure et dynamique des organisations, Paris, Eyrolles, $440 \mathrm{p}$.

Pommier, Paulette (2002). Les systèmes productifs locaux, Paris, La Documentation Française, 78 p.

Porter, Michael E. (1998). «Clusters and new economics of competition", Harvard Business Review, Vol. 76, N 6, p. 77-90.

Porter, Michael E. (2000). «Location, competition, and economic devlopment : local clusters in a global economy", Economic devlopment quarterly, Vol. 14, $\mathrm{N}^{\circ}$, p. 15-34.

Rallet, Alain; Torre, André (2004). «Proximité et localisation », Économie rurale, $\mathrm{N}^{\circ} 280$, p. 25-41.

Rychen, Frédéric; Zimmermann, Jean-Benoît (2008). «Clusters in the global knowledge-based economy: knowledge gatekeepers and temporary proximity», Regional studies, Vol. 39, $\mathrm{N}^{\circ} 1$, p. 61-74.

Tацвот, Damien (2008). «Les institutions créatrices de proximités", Revue d'Économie Régionale \& Urbaine, Vol. 2008/3, p. 289-310.

Torre, André; Rallet, Alain (2005). « Proximity and localization", Regional studies, Vol. 39, Nº 1, p. 47-59.

Tunisini, Annalisa; Bocconcelli, Roberta; Pagano, Alessandro (2011). "Is local sourcing out of fashion in the globalization era? Evidence from Italian mechanical industry? ", Industrial Marketing Management, Vol. 40, Nº, p. 1012-1023.

Vicente, Jérôme (2016). Économie des clusters, Paris, La Découverte, $128 \mathrm{p}$.

Watts, Doug H.; Wood, Andrew M.; Wardle, Perry (2006). "Owner-managers, cluster and local embeddedness : small firms in the Sheffield (UK) metal-working cluster", Entrepreneurship \& Regional Development, Vol. 18, $\mathrm{N}^{\circ} 3$, p. 185-205.

Waxell, Anders; Malmberg, Anders (2007). «What is global and what is local in knowledge-generating interaction? The case of the biotech cluster of Uppsala, Sweden », Entrepreneurship \& Regional Development, Vol. 19, N², p. 137-159.

Zimmermann, Jean-Benoît (2008). «Le territoire dans l'analyse économique : proximité géographique et proximité organisée", Revue française de gestion, Vol. 184, N²008/4, p. 105-118. 